Study on High Preci si on and Stabl e Fi ni te El ement Beam Propagat i on Net hod Based on I ncompl et e Thi rd Order Hybrid Edge/Nodal El ement

\begin{tabular}{|l|l|}
\hline 著者 & KAWAl Shohei , I GUCH Aki to, TSUJI Yasuhi de \\
\hline $\begin{array}{l}\text { j our nal or } \\
\text { publ i cat i on ti t l e }\end{array}$ & Journal of Li ght wave Technol ogy \\
\hline vol une & 36 \\
\hline number & 11 \\
\hline page range & $2278-2285$ \\
\hline year & $2018-02-28$ \\
\hline URL & ht t p: //hdl . handl e. net /10258/00009655 \\
\hline
\end{tabular}




\title{
Study on High Precision and Stable Finite Element Beam Propagation Method Based on Incomplete Third Order Hybrid Edge/Nodal Element
}

\author{
Shohei Kawai, Akito Iguchi, Student Member, IEEE, and Yasuhide Tsuji, Member, IEEE, Member, OSA
}

\begin{abstract}
It has been reported that the full vectorial finite element beam propagation method (FV-FE-BPM) is not always stable. In this paper, we propose a stabilized FV-FE-BPM using a linear filter to suppress unstable modes. Moreover, aiming for improving the computational accuracy of FV-FE-BPM for longitudinally varying optical waveguides, we employ incomplete third order hybrid edge/nodal elements to reduce interpolation error which occurs in finite element mesh update process at each propagation step. The validity and effectiveness of this approach are verified through several numerical examples of uniform waveguides and longitudinally varying waveguides.
\end{abstract}

Index Terms-Beam propagation method (BPM), finite element method (FEM), hybrid edge/nodal element

\section{INTRODUCTION}

The beam propagation method (BPM)[1] is now widely used in the analysis for longitudinally varying waveguides and various types of BPM have been proposed until now. Among them, the finite-element BPM (FE-BPM)[2]-[6] has an advantage for analyzing strongly guiding waveguides with complex cross-sectional geometry since FE-BPM can easily use an unstructured mesh which can accurately treat material boundaries. In eigenmode analysis by the full-vectorial finite element method (FV-FEM), spurious modes can be successfully suppressed by using hybrid edge/nodal elements[7] and the full-vectorial FE-BPM (FV-FE-BPM) based on LT/QN element has been also proposed[8]. However, it has been reported that the FV-FE-BPM is not always stable since unstable modes may be excited by mesh updating process at each propagation step or some numerical errors in the FV-FEBPM analysis[9],[10]. This instability is more serious in the analysis of strongly guiding waveguide. In order to overcome this instability issue, some kinds of approaches have been proposed. In the $\theta$-scheme along the propagation direction, the use of $\theta$ greater than 0.5 may suppress unstable modes, however, in this case, nonphysical attenuation may occur. In the approach based on $(p-1, p)$ Padé approximant[11], unstable modes can be suppressed depending on conditions, however, unstable modes can not be completely suppressed. Although FV-FE-BPM based on nodal elements in which rigorous interface boundary condition is treated by line integral at the boundary has been proposed [12],[13], numerical example for longitudinally varying waveguide is not demonstrated.

S. Kawai, A. Iguchi and Y. Tsuji are with the Division of Information and Electronic Engineering, Muroran Institute of Technology, Muroran, 050-8585 Japan (e-mail: 16043017@mmm.muroran-it.ac.jp; 17096001@mmm.muroran-it.ac.jp; y-tsuji@mmm.muroran-it.ac.jp).

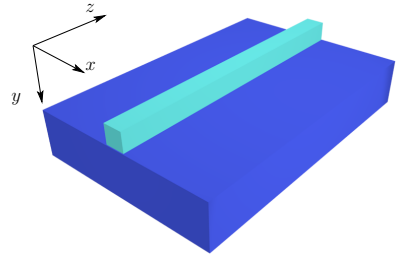

(a)

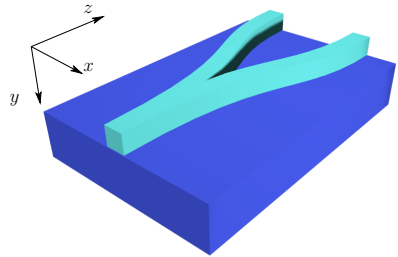

(b)
Fig. 1. 3-dimensional optical waveguides: (a) Straight waveguide and (b) Y-junction waveguide.
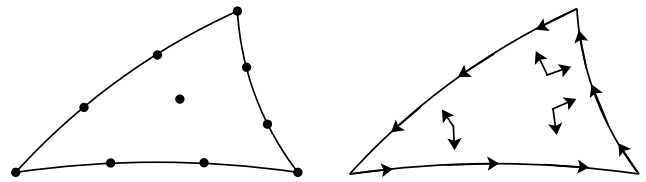

Fig. 2. Third-order incomplete hybrid edge/nodal element. The left is 3rdorder nodal element and the right is QT/CuN edge element.

In this paper, aiming at a stable analysis of longitudinally varying optical waveguides, we propose a stabilized FV-FEBPM using a linear filter[14] to suppress unstable modes. Moreover, in order to improve the computational accuracy of FV-FE-BPM, we employ higher-order hybrid edge/nodal elements QT/CuN elements[15] than LT/QN[8] elements are employed to reduce interpolation errors which occur in the finite element mesh updating process at each propagation step. To show the usefulness and effectiveness of our approach, some numerical results are shown.

\section{3 Dimensional Vector FE-BPM}

\section{A. Wave Equation}

We consider a 3-dimensional optical waveguide as shown in Fig. 1, where $x y$ and $z$ are the transverse and propagation directions respectively. From Maxwell's equations, the vectorial wave equation is expressed as follows:

$$
\boldsymbol{\nabla} \times(p \boldsymbol{\nabla} \times \boldsymbol{\Phi})-k_{0}^{2} q \boldsymbol{\Phi}=0
$$

where $k_{0}$ is the free space wavenumber and $\boldsymbol{\Phi}, p$, and $q$ are defined as follows:

$$
\begin{array}{lll}
p=1, & q=n^{2} & \text { for } \boldsymbol{\Phi}=\boldsymbol{E}, \\
p=1 / n^{2}, & q=1 & \text { for } \boldsymbol{\Phi}=\boldsymbol{H} .
\end{array}
$$

\section{B. Formulation of Eigenmode Analysis}

Dividing $x y$ cross-section of a uniform waveguide in Fig. 1(a) by hybrid edge/nodal elements in Fig. 2, and consid- 
ering light wave propagates through this structure with the propagation constant $\beta$, then the field $\boldsymbol{\Phi}$ in each element is approximated as follows:

$$
\begin{aligned}
\boldsymbol{\Phi}(x, y, z) & =\phi(x, y) \exp (-j \beta z) \\
= & {\left[\begin{array}{cc}
\{U\}^{T} & \{0\} \\
\{V\}^{T} & \{0\} \\
\{0\} & j \beta\{N\}^{T}
\end{array}\right]\left[\begin{array}{l}
\left\{\phi_{t}\right\} \\
\left\{\phi_{z}\right\}
\end{array}\right] \exp (-j \beta z) }
\end{aligned}
$$

where $\{U\},\{V\}$ are the shape functions of the edge element, $\{N\}$ is the shape function of the nodal element. Substituting (2) into (1) and applying FEM based on Galerkin method, finally, we obtain the following equation

$$
\left([K]-\beta^{2}[M]\right)\{\phi\}=\{0\}
$$

where $\{0\}$ is a null vector, and the finite element matrices $[K]$ and $[M]$, are given by

$$
[K]=\left[\begin{array}{cc}
{\left[K_{t t}\right]} & {[0]} \\
{[0]} & {[0]}
\end{array}\right], \quad[M]=\left[\begin{array}{cc}
{\left[M_{t t}\right]} & {\left[M_{t z}\right]} \\
{\left[M_{z t}\right]} & {\left[M_{z z}\right]}
\end{array}\right]
$$

with

$$
\begin{aligned}
& {\left[K_{t t}\right]=} \sum_{e} \iint_{e}\left[k_{0}^{2} q\left(\{U\}\{U\}^{T}+\{V\}\{V\}^{T}\right)\right. \\
&\left.-p\left(\frac{\partial\{V\}}{\partial x}-\frac{\partial\{U\}}{\partial y}\right)\left(\frac{\partial\{V\}^{T}}{\partial x}-\frac{\partial\{U\}^{T}}{\partial y}\right)\right] d x d y \\
& {\left[M_{t t}\right]=\sum_{e} \iint_{e}\left[p\left(\{U\}\{U\}^{T}+\{V\}\{V\}^{T}\right)\right] d x d y } \\
& {\left[M_{t z}\right]=} \sum_{e} \iint_{e}\left[p\left(\{U\} \frac{\partial\{N\}^{T}}{\partial x}+\{V\} \frac{\partial\{N\}^{T}}{\partial y}\right)\right] d x d y \\
& {\left[M_{z t}\right]=} {\left.\left[M_{t z}\right]^{T}\right] } \\
& {\left[M_{z z}\right]=} \sum_{e} \iint_{e}\left[-k_{0}^{2} q\{N\}\{N\}^{T}\right. \\
&\left.+p\left(\frac{\partial\{N\}}{\partial x} \frac{\partial\{N\}^{T}}{\partial x}+\frac{\partial\{N\}}{\partial y} \frac{\partial\{N\}^{T}}{\partial y}\right)\right] d x d y
\end{aligned}
$$

where $[0]$ is the null matrix, $\sum_{e}$ extends all the different elements.

\section{Formulation of Finite Element Beam Propagation Method}

We consider the longitudinally varying waveguide as shown in Fig. 1(b), in which light wave propagates along the $z$ direction. In the BPM analysis, the field $\boldsymbol{\Phi}$ is assumed to consist of a harmonic oscillation with the phase constant $k_{0} n_{0}$ and slowly varying envelope amplitude $\phi$. Then $\boldsymbol{\Phi}$ is approximated as follows:

$$
\mathbf{\Phi}=\phi \exp \left(-j k_{0} n_{0} z\right)
$$

where $n_{0}$ is reference refractive index. By replacing $-j \beta$ with $-j k_{0} n_{0}+\frac{d}{d z}$ in (3), the basic equation for FE-BPM is obtained as follows:

$$
\begin{aligned}
{[M] \frac{d^{2}\{\phi\}}{d z^{2}}-} & j 2 k_{0} n_{0}[M] \frac{d\{\phi\}}{d z} \\
& +\left([K]-k_{0}^{2} n_{0}^{2}[M]\right)\{\phi\}=\{0\} .
\end{aligned}
$$

Applying Padé approximation to (6), the first order partial differential equation with respect to $z$ is obtained as follows:

$$
\begin{array}{r}
-j 2 k_{0} n_{0}[\tilde{M}] \frac{d\{\phi\}}{d z}+\left([K]-k_{0}^{2} n_{0}^{2}[M]\right)\{\phi\}=\{0\} \\
{[\tilde{M}]=[M]+\frac{1}{4 k_{0}^{2} n_{0}^{2}}\left([K]-k_{0}^{2} n_{0}^{2}[M]\right) .}
\end{array}
$$

Finally, utilizing the Crank-Nicolson method along the $z$ direction, the successive updating equation of FE-BPM is given as:

$$
[A]_{k+\frac{1}{2}}\{\phi\}_{k+1}=[B]_{k+\frac{1}{2}}\{\phi\}_{k}
$$

with

$$
\begin{aligned}
& {[A]_{k}=-j 2 k_{0} n_{0}[\tilde{M}]_{k}+0.5 \Delta z\left([K]_{k}-k_{0}^{2} n_{0}^{2}[M]_{k}\right),} \\
& {[B]_{k}=-j 2 k_{0} n_{0}[\tilde{M}]_{k}-0.5 \Delta z\left([K]_{k}-k_{0}^{2} n_{0}^{2}[M]_{k}\right) .}
\end{aligned}
$$

Thus, the light propagating behavior is obtained when an incident light wave is given. In this formulation, sparsity of these matrices are maintained.

\section{EDGE/NODAl HYBRID ELEMENT}

In this section, we describe an incomplete 3rd-order edge/nodal hybrid element used in our FV-FE-BPM . In order to make our FV-FE-BPM possible to treat arbitral crosssectional geometries which include curved boundaries, the isoparametric curved elements which fit the curved boundary are employed.

\section{A. $Q T / C u N$ edge element}

The shape functions of the 3rd-order edge element used in this study are expressed as follows:

$$
\begin{aligned}
\boldsymbol{N}_{t, 3 i-2} & =\frac{1}{2}\left(L_{i} \nabla L_{j}-L_{j} \nabla L_{i}\right)\left(4 L_{i}-1\right)\left(4 L_{i}-2\right) \\
\boldsymbol{N}_{t, 3 i-1} & =\left(L_{i} \nabla L_{j}-L_{j} \nabla L_{i}\right)\left(4 L_{i}-1\right)\left(4 L_{j}-1\right) \\
\boldsymbol{N}_{t, 3 i} & =\frac{1}{2}\left(L_{i} \nabla L_{j}-L_{j} \nabla L_{i}\right)\left(4 L_{j}-1\right)\left(4 L_{j}-2\right) \\
\boldsymbol{N}_{t, 2 i+8} & =\left(L_{j} \nabla L_{k}-L_{k} \nabla L_{j}\right) L_{i}\left(4 L_{i}-1\right) \\
\boldsymbol{N}_{t, 2 i+9} & =\left(L_{k} \nabla L_{i}-L_{i} \nabla L_{k}\right) L_{j}\left(4 L_{i}-1\right)
\end{aligned}
$$

where $(i, j, k)=(1,2,3),(2,3,1),(3,1,2)$ and $L_{i}$ is the area coordinate. In the $\mathrm{QT} / \mathrm{CuN}$ edge element, the tangential and normal components of an edge are interpolated by a 2nd-order and 3rd-order polynomials, respectively. Since the interpolation accuracy of the field distribution in BPM analysis is more important than in the guided mode analysis, utilizing the QT/CuN edge element based on 2nd or higher-order interpolation for all the components can effectively reduce the interpolation error during mesh updating process which is required at each propagation step.

\section{B. Nodal Element}

The shape functions of 3rd order nodal elements used in this study are expressed as follows:

$$
\begin{aligned}
\boldsymbol{N}_{i} & =\frac{1}{2} L_{i}\left(3 L_{i}-1\right)\left(2 L_{i}-2\right) \\
\boldsymbol{N}_{2 i+2} & =\frac{9}{2} L_{i} L_{j}\left(3 L_{i}-1\right)
\end{aligned}
$$




$$
\begin{aligned}
\boldsymbol{N}_{2 i+3} & =\frac{9}{2} L_{i} L_{j}\left(3 L_{j}-1\right) \\
\boldsymbol{N}_{10} & =27 L_{1} L_{2} L_{3}
\end{aligned}
$$

where $(i, j)=(1,2),(2,3),(3,1)$.

\section{Isoparametric Curved Element}

In order to discretize the waveguide cross-section with curved material boundaries, we use the isoparametric curved element whose boundary is approximated by the same order polynomials as those for field distribution[16].

Using the shape function $\{N\}$, the coordinates in triangular elements are approximated by

$$
x=\{N\}^{T}\{x\}_{e}, \quad y=\{N\}^{T}\{y\}_{e}
$$

where $\{x\}_{e}$ and $\{y\}_{e}$ are the vectors which consist of the coordinates at all nodes in the element. Using the Jacobian matrix $[J]$, the conversion formula for the differential operators are shown as follows:

$$
\left[\begin{array}{l}
\frac{\partial}{\partial L_{1}} \\
\frac{\partial}{\partial L_{2}}
\end{array}\right]=[J]\left[\begin{array}{l}
\frac{\partial}{\partial x} \\
\frac{\partial}{\partial y}
\end{array}\right]=\left[\begin{array}{ll}
J_{11} & J_{12} \\
J_{21} & J_{22}
\end{array}\right]\left[\begin{array}{l}
\frac{\partial}{\partial x} \\
\frac{\partial}{\partial y}
\end{array}\right]
$$

with

$$
\begin{array}{ll}
J_{11}=\frac{\partial\{N\}^{T}}{\partial L_{1}}\{x\}_{e}, & J_{12}=\frac{\partial\{N\}^{T}}{\partial L_{1}}\{y\}_{e}, \\
J_{21}=\frac{\partial\{N\}^{T}}{\partial L_{2}}\{x\}_{e}, & J_{22}=\frac{\partial\{N\}^{T}}{\partial L_{2}}\{y\}_{e} .
\end{array}
$$

\section{STABILITY OF FV-FE-BPM}

In this section, we consider lossless waveguides to discuss the stability of FV-FE-BPM. The eigenmodes of a uniform waveguide are obtained by solving (3). Considering the $i$-th eigenmode at $k$-th propagation step, whose eigenvector and propagation constant are $\left\{\phi_{i}\right\}_{k}$ and $\beta_{i}$, respectively, the field vector at $(k+1)$-th step, $\left\{\phi_{i}\right\}_{k+1}$ is expressed as follows:

$$
\begin{aligned}
\left\{\phi_{i}\right\}_{k+1} & =\frac{-j 2 k_{0} n_{0}-0.5 \Delta z\left(\beta_{i}^{2}-k_{0}^{2} n_{0}^{2}\right)}{-j 2 k_{0} n_{0}+0.5 \Delta z\left(\beta_{i}^{2}-k_{0}^{2} n_{0}^{2}\right)}\left\{\phi_{i}\right\}_{k} \\
& =A_{i, k}\left\{\phi_{i}\right\}_{k} .
\end{aligned}
$$

Because of the conservation law, the total transmission power across the waveguide cross-section at $(k+1)$-th step in FEBPM has to be equal to that at $k$-th step. However, we can see that the amplification coefficient, $A_{i, k}$, depends on the eigenvalue, $\beta_{i}^{2}$. If $\beta_{i}^{2}$ is purely real, the absolute value of $A_{i, k}$ is equal to 1 and the power conservation law is satisfied since the denominator and the numerator are complex conjugate with each other. In the case of $2 \mathrm{D}$ analysis or scalar approximated analysis for 3D waveguides, all the eigenvalues are real and the power conservation always holds. However, in the full vectorial analysis for 3D waveguides, it is known that some eigenvalues have imaginary parts and the power conservation is not always satisfied. (14) can be rewritten as follows:

$$
\begin{gathered}
\left\{\phi_{i}\right\}_{k+1}=\frac{a-j b^{+}}{-a-j b^{-}}\left\{\phi_{i}\right\}_{k}, \\
a=\frac{\Delta z}{2}\left(\operatorname{Re}\left\{\beta_{i}^{2}\right\}-k_{0}^{2} n_{0}^{2}\right), \quad b^{ \pm}=2 k_{0} n_{0} \pm \frac{\Delta z}{2} \operatorname{Im}\left\{\beta_{i}^{2}\right\} .
\end{gathered}
$$

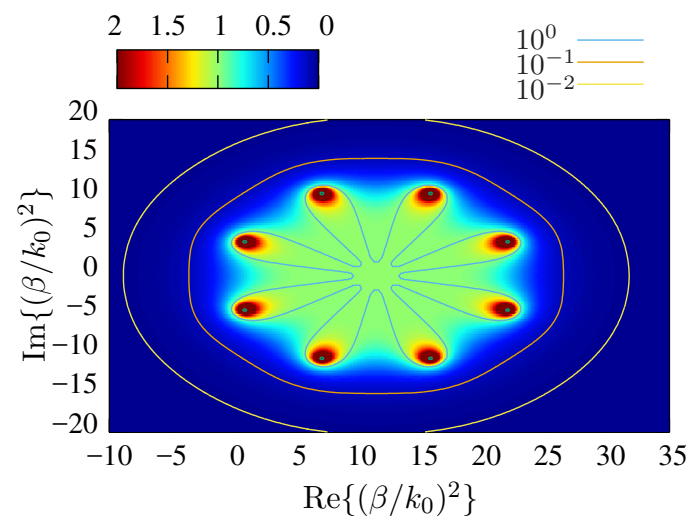

(a)

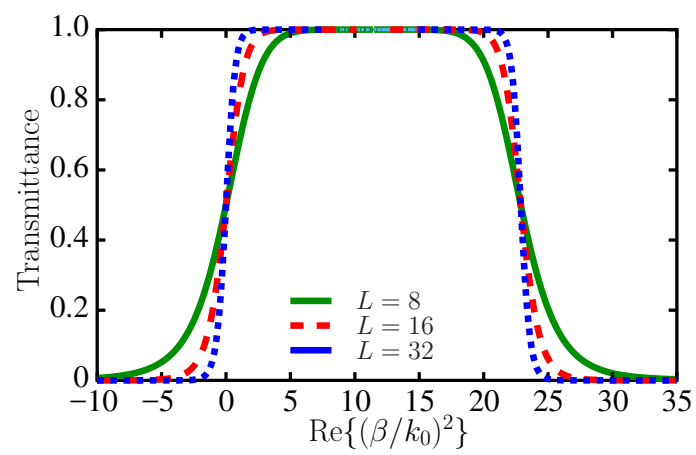

(b)

Fig. 3. The transmission property of linear filter $(L=8)$ : (a) The transmission property on complex plane and (b) The transmission property on the real axis.

In this case, the denominator and the numerator are no longer complex conjugate with each other since $b^{-} \neq b^{+}$. Thus, the power conservation is not satisfied for these complex modes. When $\operatorname{Im}\left\{\beta^{2}\right\}<0$ and $b^{+}<b^{-}$, the amplification coefficient is smaller than 1 and it causes unwanted power loss. On the other hand, when $\operatorname{Im}\left\{\beta^{2}\right\}>0$ and $b^{+}>b^{-}$, the amplification coefficient is greater than 1 and it may cause instability of a propagating field.

\section{Stabilization of FV-FE-BPM by Linear FiLter}

In this section, we describe an approaches to stabilize FVFE-BPM solutions using a linear filter. For simplicity, we consider a linear filter constructed by FEM matrices, $[M]$ and $[K]$, used in BPM analysis although there may be several approaches to construct a linear filter. Here, each eigenmode derived from (3) satisfies the following relation:

$$
\left([K]-\beta_{l}^{2}[M]\right)^{-1}[M]\left\{\phi_{i}\right\}=\frac{1}{\beta_{i}^{2}-\beta_{l}^{2}}\left\{\phi_{i}\right\}
$$

where $\beta_{l}$ is a value to be specified for constructing a desired linear filter. This operation on arbitrary propagating field amplify the eigenvector components around $\beta_{l}$ and suppress those far from $\beta_{l}$. Utilizing this operator, we are able to 


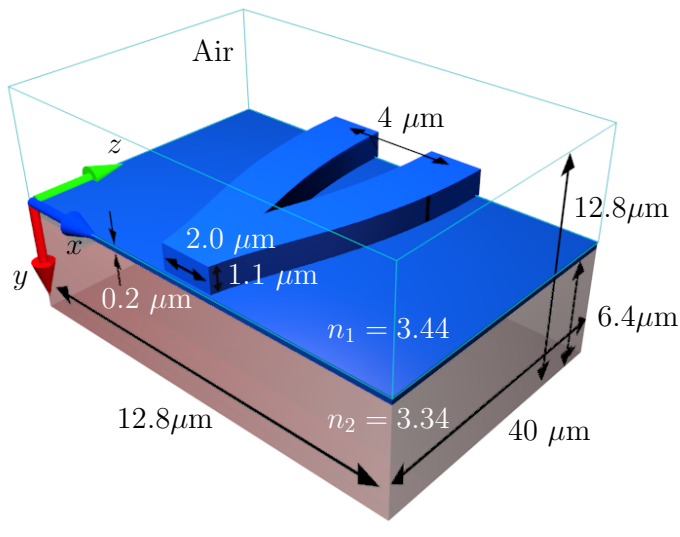

Fig. 4. 3-dimensional Y-junction optical rib waveguide.

construct the following linear filter:

$$
\begin{aligned}
F\left(\beta^{2}\right) & =\prod_{l=1}^{L} \frac{a[M]}{[K]-\left(a t_{l}+t_{0}\right)[M]} \\
t_{l} & =e^{j(2 l-1) \pi / L}
\end{aligned}
$$

where $\left(t_{0}, 0\right)$ is the specified central complex value of the band pass filter on the complex propagation constant plane, $a$ is the filter radius, and $L$ is the order of the filter. The transmission property of this filter with $L=8$ is shown in Fig. 3. In the usual BPM analysis, only the field components with positive real eigenvalues are able to be treated and the other field components cause instability or degradation of numerical accuracy. Thus, we would like to realize a linear filter which passes only the field components with positive real eigenvalues. From Fig. 3, we can see that this filter is able to pass the eigenmodes within the radius $a$ from the center $\left(t_{0}, 0\right)$. It is known that the eigenmodes expressed by FV-FEM based on hybrid edge/nodal element do not have complex modes in the positive real eigenvalue space. As a result, utilizing this filter, we can effectively eliminate the eigenmode components with complex eigenvalues. The transmission property of this filter on the real axis $(L=8,16,32)$ is shown in Fig. 3(b). We can see that the modes to be considered in the BPM analysis hardly attenuate if the filter parameters are appropriately selected. Although the filter parameters has to be appropriately selected depending on problems, those can be easily determined by the maximum refractive index $n_{\max }$ among all materials in the computational domain since the effective indices of the modes to be treated in BPM lie in the range between 0 and $n_{\max }$. It is shown that the numerical results do not severely depend on the filter parameters in the next section.

\section{FV-FE-BPM with LINEAR FILTER}

In order to confirm the effectiveness of the proposed filter, we consider a Y-branching waveguide shown in Fig. 4 and simulate the propagation behavior by the FV-FE-BPM with the proposed linear filter $\left(L=16, t_{0}=0.75 \beta_{0, \text { in }}^{2}, a=0.75 t_{0}\right.$, where $\beta_{0, \text { in }}$ is the propagation constant of the input mode). The operating wavelength is set to be $1.55 \mu \mathrm{m}$ and the perfectly matched layer (PML) is used as an absorbing boundary
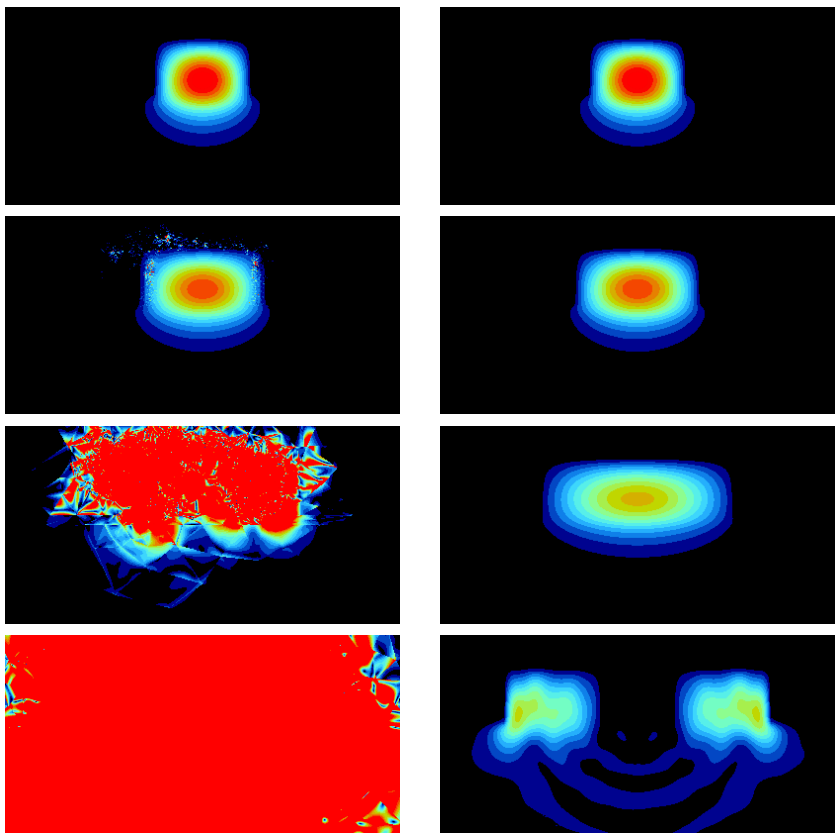

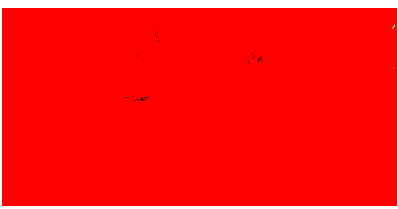

(a)

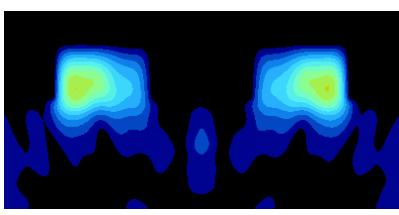

(b)
Fig. 5. Magnetic field amplitude at each propagation step (In order from the top, $z=0,10,20,30$, and $40 \mu \mathrm{m}$ ): (a) Conventional FV-FE-BPM and (b) FV-FE-BPM with a linear filter.

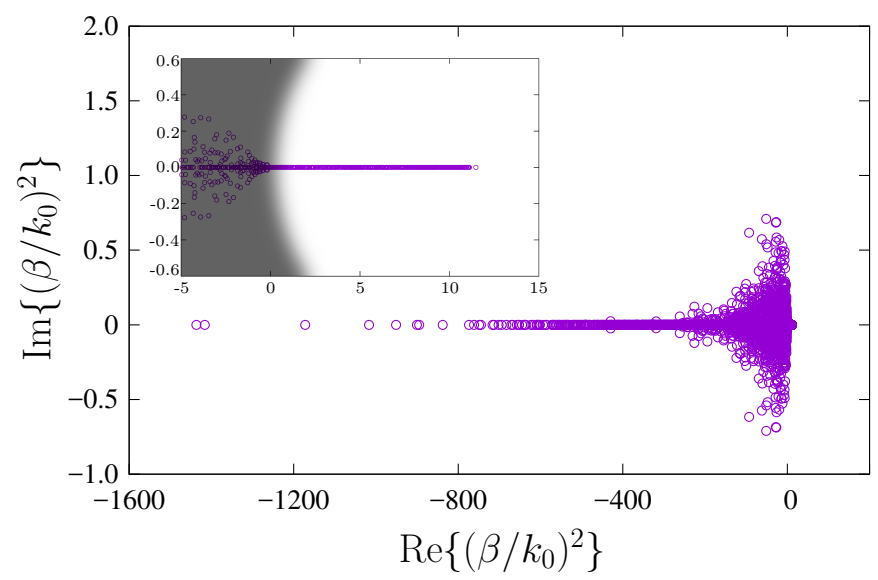

Fig. 6. Eigenmode spectrum of the input waveguide as shown in Fig. 4. The inset is an enlarged view around $\left(\beta / k_{0}\right)^{2}=0$.

condition in the FV-FE-BPM analysis. The PML may cause instability of FV-FE-BPM because the eigenmode spectrum is changed and eigenmodes may appear in the vicinity of the filter poles shown in Fig. 3(a). In the following discussion, in order to avoid unexpected instability, the FEM matrices in (16) are constructed without using PML boundary condition. The PML is applied only to the field updating equation (8). The variation along the $z$-direction of the center of the waveguide is set to be $x= \pm\{1-\cos (\pi z / 40 \mu \mathrm{m})\}$. In this analysis, the finite element mesh is adaptively updated according to 


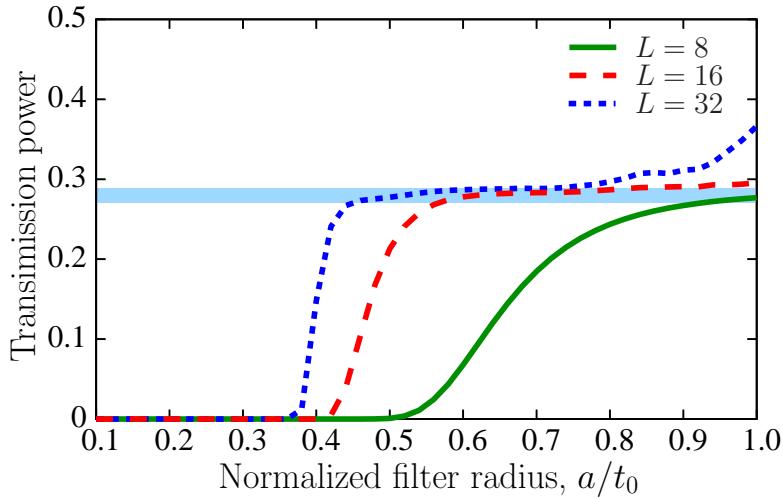

Fig. 7. Normalized transmission power of fundamental mode at each port as a function of filter radius $\left(t_{0}=0.75 \beta_{0, \text { in }}^{2}\right)$

the waveguide geometry and propagating field distribution at each propagation step and about 5,000 QT/CuN elements on average are used to discretize waveguide cross-section. The light propagating behavior at each propagation step is shown in Fig. 5. For comparison, the results without the linear filter are also shown. We can see that the instability is effectively suppressed by using the linear filter, while the propagating field is unstable in the result without the filter. Figure 6 shows the eigenvalue distribution of the input waveguide obtained by FV-FEM. The inset is the enlarged view around $\beta^{2}=0$. The modes of $\operatorname{Im}\left\{\beta^{2}\right\}>0$ are the unstable modes. However, in the region where $\operatorname{Re}\left\{\beta^{2}\right\}>0$, unstable modes do not exist and the filter used here is able to pass only stable modes. In the enlarged view, the schematic image of filtering process is also shown. The modes in the shaded region are suppressed at each propagation step. However, in this approach, it seems that the filter radius has to be carefully selected. If the filter radius is too large, unstable mode may not be completely suppressed, and if the filter radius is too small, unexpected propagation loss occurs. In order to make this issue clear, we calculate the fundamental mode power at the output end for several filter parameters. Figure 7 shows the normalized output power of the fundamental mode in each output port as a function of the normalized filter radius $a / t_{0}$. Around $a / t_{0}=0.65$, the calculated output power is almost independent of the filter radius in the case of filter order $L=16$ and 32 . On the other hand, in the case of $L=8$, the unwanted modes are not able to be suppressed or undesired propagation loss occurs because of insufficient filter sharpness. When the filter order is higher, the calculated accuracy degrades faster as the filter radius is larger. This is because the higher order filter has more poles than the lower order one. From these results, we can see that the numerical results are not sensitively affected by the selection of the filter parameters if we select $a / t_{0}$ of around 0.65 .

\section{ACCURACY OF HIgHER-ORdER EdGE/NODAL HYBRID ELEMENT}

In this section, we investigate the numerical accuracy of the full-vectorial analysis using curvilinear hybrid edge/nodal elements in the view of eigenvalues and interpolated field

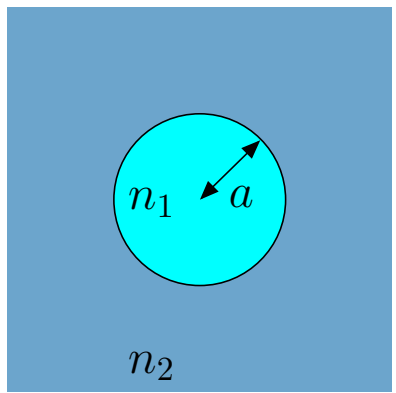

Fig. 8. Optical fiber with high refractive index contrast.

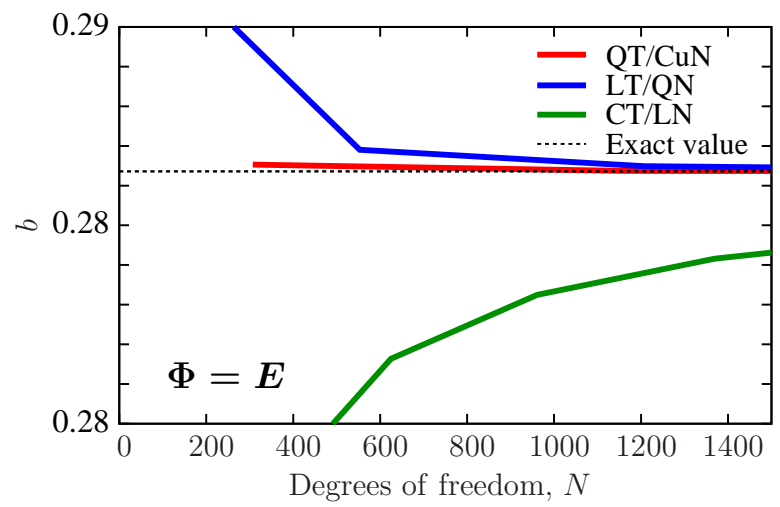

(a)

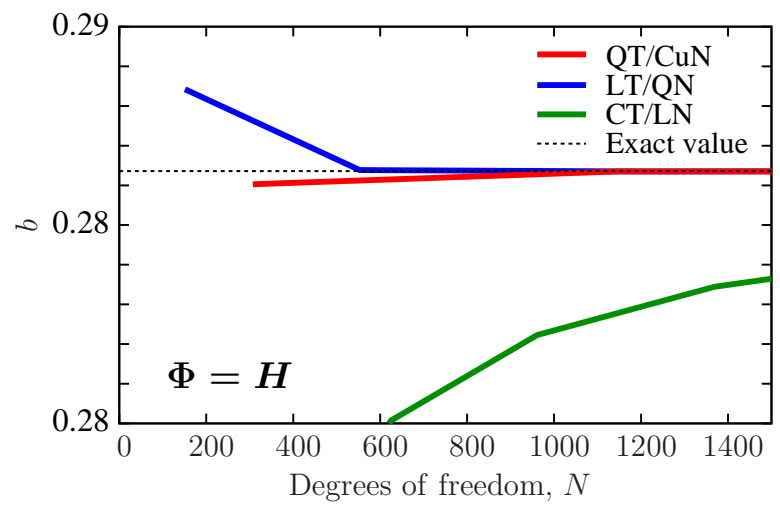

(b)

Fig. 9. Convergence of the effective refractive index of $\mathrm{HE}_{11}^{x}$ mode in the optical fiber for (a) $\Phi=\boldsymbol{E}$ and (b) $\Phi=\boldsymbol{H}$.

distributions since the interpolation of the field at each step is an essential issue in the FE-BPM analysis. CT/LN, LT/QN, and QT/CuN edge elements are discussed and compared with each other.

\section{A. Numerical Accuracy of Eigenmode Calculation}

In order to discuss the numerical accuracy of FV-FEM with different order elements, we consider the ultra-high refractive index contrast optical fiber shown in Fig. 8. The refractive indices in the core and cladding are assumed to be $n_{1}=1.515$ and $n_{2}=1$, respectively, and the normalized frequency is assumed to be $v=2$. In this example, the approximation of curved boundary is also able to be discussed. Figure 9 shows the convergence behavior of the calculated 

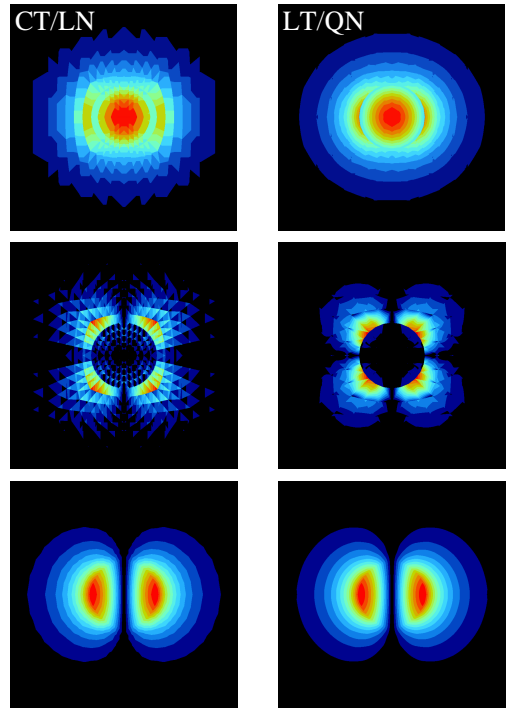

(a)

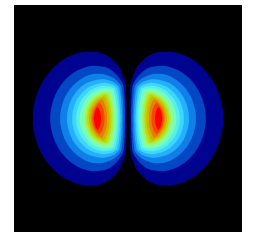

(b)
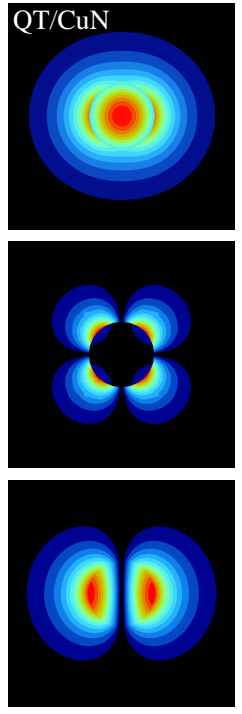

(c)
Fig. 10. The mode field distribution of the $\mathrm{HE}_{11}^{x}$ mode of the optical fiber. (In order from the top, $E_{x}, E_{y}$ and $\left.E_{z}\right)$. (a) $\mathrm{CT} / \mathrm{LN}(N=961)$, (b) LT/QN $(N=$ 1207) and (c) QT/CuN $(N=1153)$.

normalized propagation constant $b$ of $\mathrm{HE}_{11}^{x}$ mode depending on the degrees of freedom $N$ in the eigenmode analysis by FV-FEM. From this figure, we can see that the LT/QN and QT/CuN elements bring us higher accuracy with fewer elements than CT/LN elements. We can also see that the numerical accuracy of LT/QN and QT/CuN elements is not so different in the view of eigenvalues. On the other hand, in the view of approximated field distributions, the accuracy may not be sufficient in the LT/QN element. Figure 10 shows the approximated field distributions obtained by using different order elements. In this figure, the degrees of freedom used in FEM analysis is selected to be almost same. Compared with these field distributions, we can see that the interpolated field distribution by LT/QN elements has unexpected distortion and the interpolation accuracy is insufficient compared with QT/CuN element, especially in the region where the field intensity is small.

\section{B. Numerical Accuracy of Field Interpolation}

In order to demonstrate the influence of the field interpolation on the FV-FE-BPM analysis, the transmission power is calculated for the numerical example shown in Fig. 4, using CT/LN, LT/QN, and QT/CuN elements. Figure 11 shows the input mode field profile in the case that the degrees of freedom is $N=2257$ for $\mathrm{CT} / \mathrm{LN}, N=3489$ for LT/QN, and $N=1897$ for QT/CuN, respectively. In this modal analysis, the half region of the whole structure is analyzed utilizing the structural symmetry. As we can see in these figures, the interpolation accuracy of the lower order (CT/LN and LT/QN) elements is not sufficient. This interpolation error may become considerable numerical error since the numerical error is accumulated in the BPM analysis. Figure 12 shows the normalized transmission power at the output end as a function of degrees of freedom. The total power including guided modes and radiated modes shown in Fig. 12(a) and

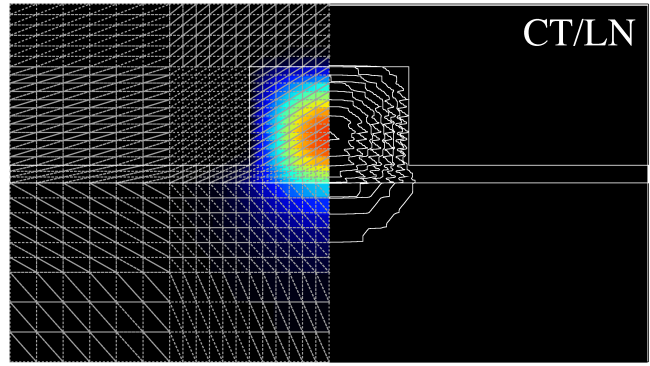

(a)

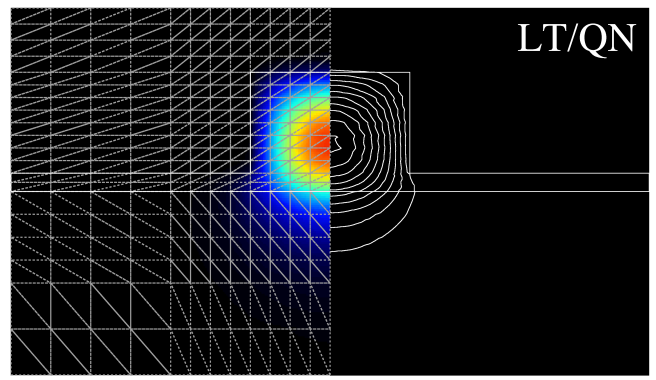

(b)

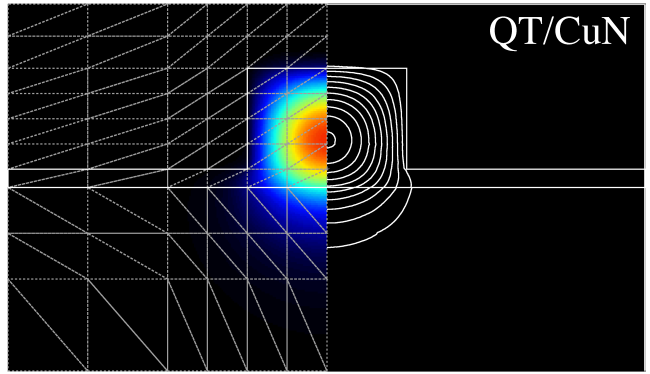

(c)

Fig. 11. The mode field distribution $\left(H_{y}\right)$ of $E_{11}^{x}$ mode and finite element mesh at the input end: (a) $\mathrm{CT} / \mathrm{LN}(N=2257)$, (b) $\operatorname{LT} / \mathrm{QN}(N=3489)$ and (c) $\mathrm{QT} / \mathrm{CuN}(N=1897)$.

the fundamental mode power at each port is shown in Fig. 12(b). The normalized transmission power of the fundamental mode is 0.281 (converged value) at each port. This is in good agreement with results shown in [4]. We can see that the convergence speed is much faster for the QT/CuN elements than for the LT/QN elements, and computational cost is greatly reduced by using higher order QT/CuN elements.

\section{ApPlication to Plasmonic DeVices}

In order to confirm the applicability of the proposed FV-FEBPM to plasmonic devices, we consider the surface plasmon stripe waveguide (SPWG) shown in Fig. 13 and discuss the numerical accuracy of our FV-FE-BPM in lossy and leaky waveguide analysis. The waveguide parameters are the same as in [17]. The refractive indices of Au stripe and fused quartz substrate are assumed to be $\varepsilon_{m}=-26.1437-j 1.8497$ and $\varepsilon_{s}=1.46^{2}$, respectively. The stripe height is $h=55 \mathrm{~nm}$ and the operating wavelength is set to be $0.8 \mu \mathrm{m}$. The computational domain is surrounded by the PML to absorb radiated wave. Figure 14 shows the effective refractive indices of the leaky modes as a function of the stripe width $W$. 


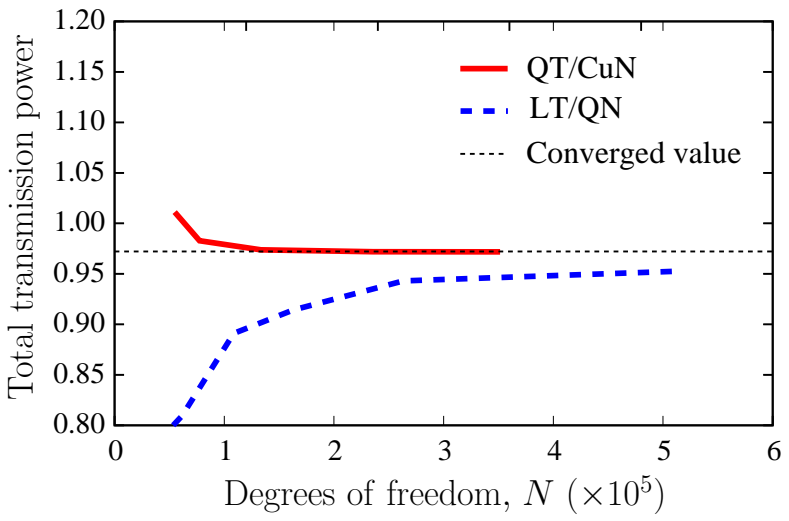

(a)

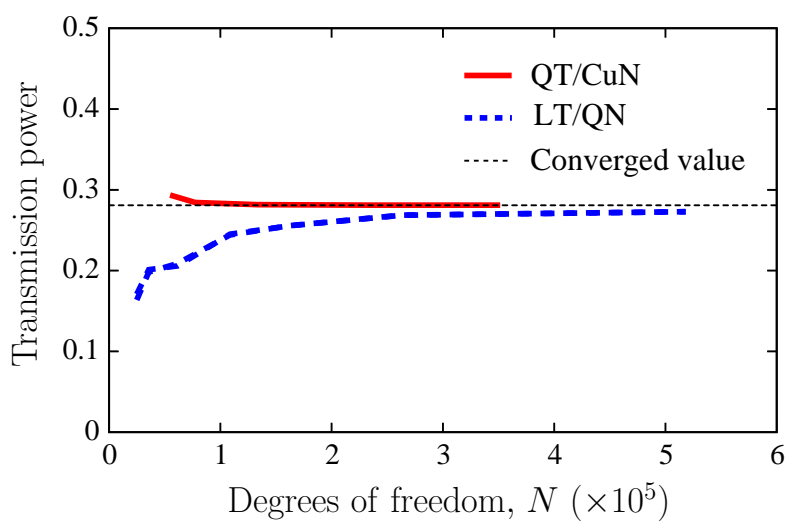

(b)

Fig. 12. Normalized transmission power at output end as a function of degrees of freedom. (a) all modes, (b) fundamental mode at each port.

These results by our FV-FEM are in good agreement with those in [17]. Figure 15 shows the propagation behavior in the SPWG and Fig. 16 shows the normalized transmission power in the computational domain as a function of propagation distance. In this calculation, the stripe width $W$ is set to be $3.7 \mu \mathrm{m}$ and the filter parameters are set as follows: $L=16$, $t_{0}=0.75 \beta_{0, \text { in }}^{2}$, and $a=0.75 t_{0}$. The theoretical curve obtained by the eigenmode analysis is also shown in Fig. 16. According to Fig.15 and Fig 16, we can see that unwanted instability does not occur and the fields are attenuated along the propagation direction. According to Fig.16, we can also see that the spurious attenuation originated from the proposed filter does not occur because the attenuation rate obtained from the results of the FV-FE-BPM analysis is in very good agreement with the theoretical value. From these results, the potential of applicability to plasmonic devices is shown and the numerical accuracy of our FV-FE-BPM is confirmed even for lossy and leaky waveguides.

\section{CONCLUSION}

In this paper, we proposed the stabilization approach of FVFE-BPM based on a linear filter to suppress unwanted complex modes. The proposed approach is able to stabilize FV-FEBPM by selecting the appropriate filter radius. Moreover, we investigated the numerical accuracy of the FV-FE-BPM when

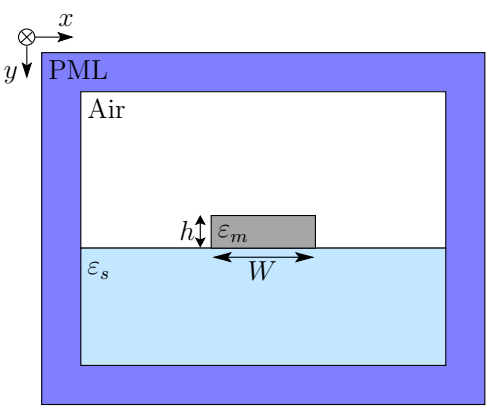

Fig. 13. Surface plasmon stripe waveguide.

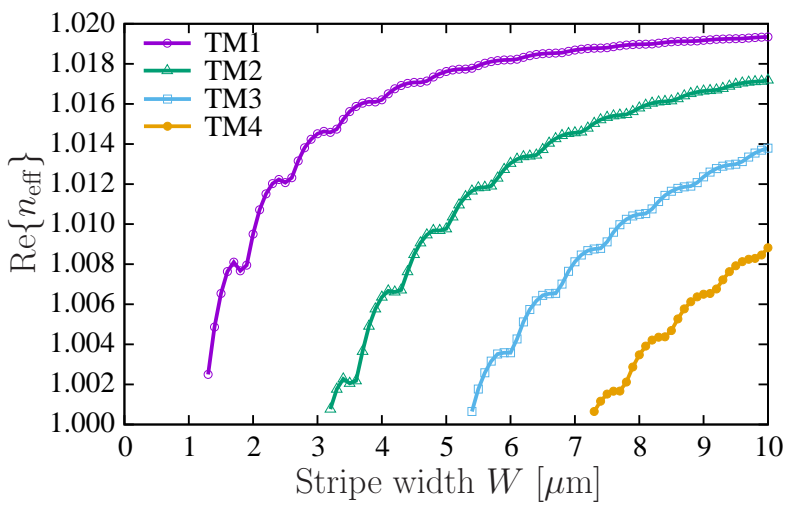

(a)

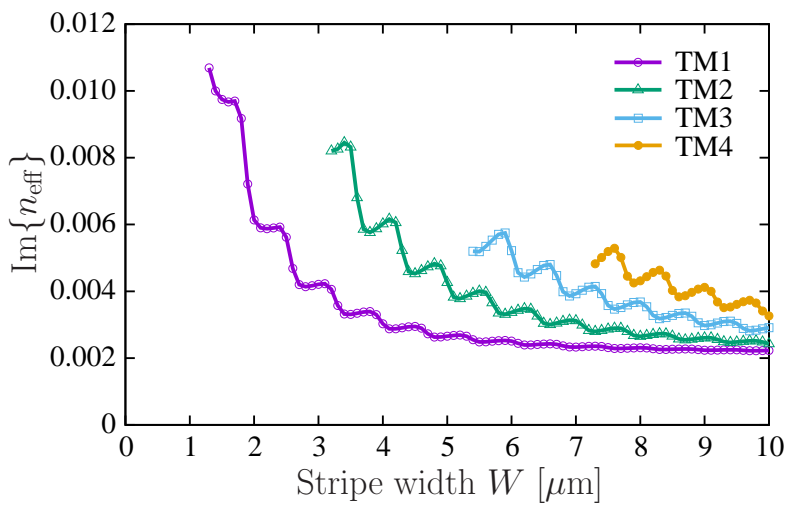

(b)

Fig. 14. Effective refractive index $n_{\text {eff }}$ of leaky modes as a function of stripe Au width $W$. (a) real part of $n_{\text {eff }}$, (b) imaginary part of $n_{\text {eff }}$.

different order elements were used in detail. The simulation results demonstrated that the interpolation accuracy of the field distribution is dramatically improved by using higher order elements and computational cost can be greatly reduced, even in the case that the computational accuracy of the eigenvalues is not so different between LT/QN and QT/CuN elements. Nowadays, the importance of photonic devices with high refractive index difference is increasing. In order to treat such devices by BPM, bidirectional extension or other improvement is required and that is our future work.

\section{ACKNOWLEDGEMENT}

This work was supported by JSPS KAKENHI Grant Number $15 \mathrm{~K} 06009$. 

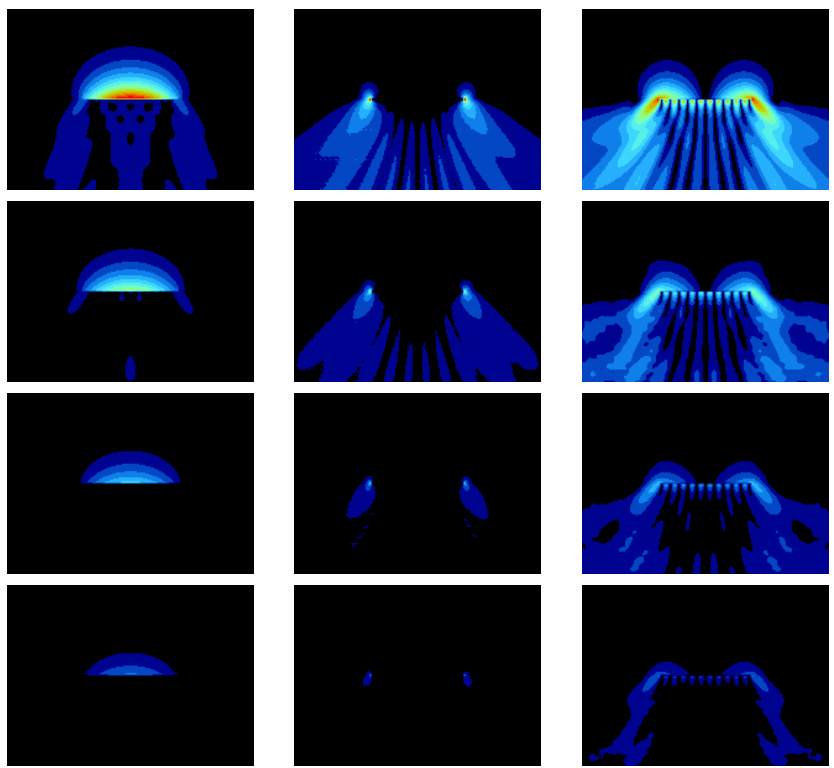

(a)

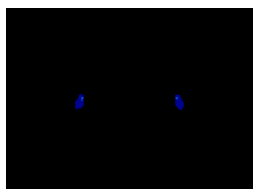

(b)
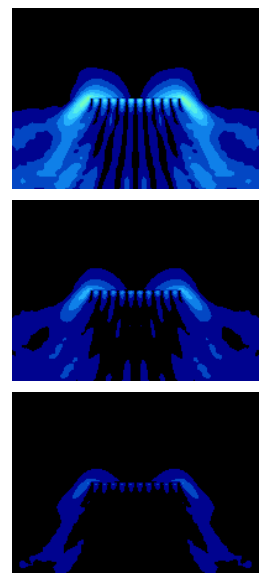

(c)

Fig. 15. Magnetic field amplitude of the SPWG at each propagation step (In order from the top, $z=0,20,40$ and $60 \mu \mathrm{m}$ ). (a) $H_{x}$, (b) $H_{y}$ and (c) $H_{z}$.

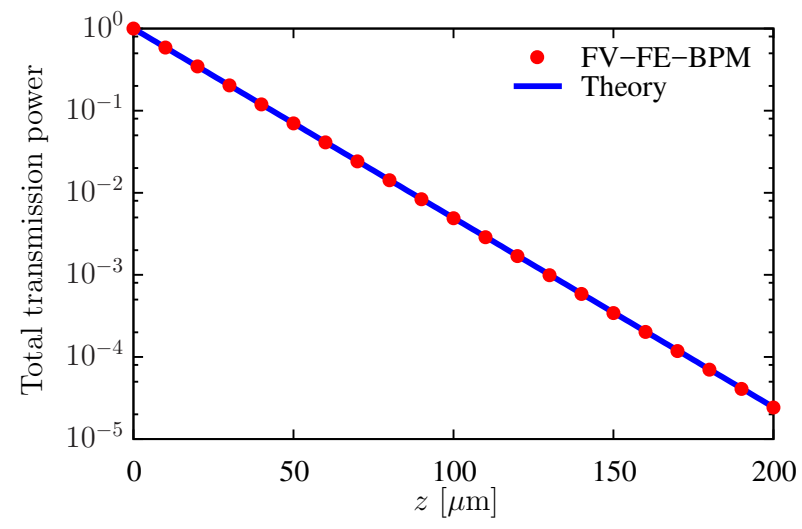

Fig. 16. Normalized total transmission power of SPWG at each propagation step.

\section{REFERENCES}

[1] M. D. Feit and J. A. Fleck, Jr., "Light propagation in graded index optical fibers," Appl. Opt., vol. 17, no. 24, pp.3990-3998, Dec. 1978.

[2] Y. Tsuji and M. Koshiba, "A finite element beam propagation method for strongly guiding and longitudinally varying optical waveguides," $J$. Lightw. Technol., vol. 14, no. 2, pp. 217-222, Feb. 1996.

[3] Y. Tsuji and M. Koshiba, "Finite element beam propagation method for three-dimensional optical waveguide structures," J. Lightw. Technol., vol. 15, no. 9, pp. 1728-1734, Sep. 1997.

[4] E. E. Kriezis and A. G. Pagagiannakis, "A three-deimensional full vectorial beam propagation method for z-dependent structures," J. Quantum Electoron., vol. 33, no. 5, pp. 883-890, May 1997.

[5] D. Schulz, C. Gingener, M. Bludsuweit, and E. Voges, "Mixed finite element beam propagation method," J. Lightw. Technol., vol. 16, no. 7, pp. 1336-1341, July 1998.

[6] E. Montanari, S. Selleri, L. Vincetti, and M. Zoboli, "Finite-element full-vectorial propagation analysis for three-dimensional z-varying optical waveguides," J. Lightw. Technol., vol. 16, no. 4, pp. 703-714, Apr. 1998.

[7] M. Koshiba and K. Inoue, "Simple and efficient finite-element analysis of microwave and optical wavegides," IEEE Trans. Microwave Theory Tech., vol. 40, pp. 371-377, Feb. 1992.

[8] Y. Tsuji and M. Koshiba, "Adaptive mesh generation for full-vectorial guided-mode and beam-propagation solutions," IEEE J. Sel. Top. Quantum Electron., vol. 6, no. 1, pp. 163-169, Jan. 2000.

[9] H. Deng and D. Yevick, "The nonunitarity of finite-element beam propagation algorithms," IEEE Photon. Technol. Lett., vol. 17, no. 7, pp. 1429-1431, July 2005.

[10] T. Q. Tran and S. Kim, "Stability condition of finite-element beam propagation methods in lossy waveguides," IEEE J. Quantum Electron., vol. 50, no. 10, pp. 808-814, Oct. 2014.

[11] S. L. Chui and Y. Y. Lu, "A propagator- $\theta$ beam propagation method," IEEE Photon. Technol. Lett., vol. 16, no. 3, pp. 822-824, Mar. 2004.

[12] S. S. A. Obayya, B. M. A. Rahman, and H. A. El-Mikati, "New fullvectorial numerical efficient propagation algorithm based on the finite element method," J. Lightw. Technol., vol. 18, no. 3, pp. 409-415, Mar. 2000.

[13] S. S. A. Obayya, B. M. A. Rahman, and H. A. El-Mikati, "Full-vectorial finite-element beam propagation method for nonlinear directional coupler devices," IEEE J. Quantum Electron., vol. 36, no. 5, pp. 556-562, May 2000.

[14] H. Murakami, "A filter diagonalization method by the linear combination of resolvents," IPSJ Trans. Advanced Computing Systems, vol. 49, no. SIG 2 (ACS 21), pp. 66-87, Mar. 2008.

[15] R. D. Graglia, D. R. Wilton, and A. F. Peterson, "Higher order interpolatory vector bases for computational electromrgnetics," IEEE Trans. Antennas Propagat., vol. 45, no. 3, pp. 329-342, Mar. 1997.

[16] M. Koshiba and Y. Tsuji, "Curvilinear hybrid edge/nodal elements with triangular shape for guidedwave problems," J. Lightw. Technol., vol. 18, no. 5, pp. 737-743, May 2000.

[17] H. H. Liu and H. C. Chang, "High-resolution analysis of leaky modes in surface plasmon stripe waveguide," J. Lightw. Technol., vol. 34, no. 11, pp. 2752-2757, June 2016.

Shohei Kawai received the B.S. degree in information and electronic engineering from Muroran Institute of Technology, Muroran, Japan, in 2016. He is presently studying toward the M.S. degree in information and electronic engineering at Muroran Institute of Technology.

Mr. Kawai is a student member of the Institute of Electronics, Information and Communication Engineers (IEICE).

Akito Iguchi (S'16) received the B.S. and M.S. degrees in information and electronic engineering from Muroran Institute of Technology, Muroran, Japan, in 2015 and 2017, respectively. He is presently studying toward the Ph.D. degree in information and electronic engineering at Muroran Institute of Technology.

Mr. Iguchi is a student member of the Institute of Electronics, Information and Communication Engineers (IEICE) and IEEE.

Yasuhide Tsuji (M'97) received the B.S., M.S., and Ph.D. degrees in electronic engineering from Hokkaido University, Sapporo, Japan, in 1991, 1993, and 1996, respectively.

In 1996, he joined the Department of Applied Electronic Engineering, Hokkaido Institute of Technology, Sapporo, Japan. From 1997 to 2004, he was an Associate Professor of Electronics and Information Engineering at Hokkaido University. From 2004 to 2011, he was an Associate Professor of Electrical and Electronic Engineering at Kitami Institute of Technology, Kitami, Japan. Since 2011, he has been a Professor of Information and Electronic Engineering at Muroran Institute of Technology, Muroran, Japan. $\mathrm{He}$ has been interested in wave electronics.

Dr. Tsuji is a member of the Institute of Electronics, Information and Communication Engineers (IEICE), the Japan Society of Applied Physics, the Optical Society of America (OSA), and IEEE. In 1997 and 1999, he was awarded the Excellent Paper Award from IEICE. In 2000, he has received the Third Millennium Medal from IEEE. 